Revista de Derecho

Universidad Católica del Norte

Sección: Ensayos

Año 17 - No 2, 2010

pp. $167-183$

\title{
REFLEXIONES SOBRE LAS EVOLUCIONES RECIENTES EN MATERIA DE PROTECCIÓN DE RIESGOS SOCIALES A NIVEL INTERNACIONAL ${ }^{*}$
}

\author{
PABlo ANDrÉs AREllano OrTiz ${ }^{* *}$
}

RESUMEN: El presente trabajo pretende destacar distintos niveles de cobertura de los riesgos sociales, mostrando sus evoluciones y problemas. Se analiza el Estado de bienestar y la seguridad social como mecanismos tradicionales de cobertura analizando sus deficiencias desde un punto de vista comparado. A estos dos niveles se agrega el piso de protección social que pretende ser consagrado por la OIT para fortalecer los dos niveles antes mencionados. Se realiza una crítica a este último en función de su carácter no normativo y desconexión de los otros dos niveles.

PALABRAS CLAVE: Seguridad social - derechos humanos - OIT piso social - Convenio $\mathrm{N}^{\circ} 102$.

\section{REFLECTING ABOUT THE RECENT EVOLUTION REGARDING SOCIAL RISKS AT AN INTERNATIONAL LEVEL}

ABSTRACT: This piece of work intends to highlight different levels of coverage of the social risks, showing its evolution and problems. The Welfare state concept and Social Security are analyzed as traditional mechanisms of coverage indicating its deficiencies from a comparative point of view. These two levels are added one of social protection that intends to be established by the ILO to strengthen the two former levels. We find a critique to this social protection level regarding its nonnormative character and to its disconnection to the other two levels.

El autor agradece los comentarios en versiones preliminares de este trabajo de Fabio Duran Valverde (SecSoc -OIT, Ginebra) y de Sergio Gamonal Contreras (Universidad Adolfo Ibáñez, Chile), así como también las sugerencias constructivas de los dos relectores anónimos.

** Doctor en Derecho por la Université Paris Ouest Nanterre La Defense (Francia). Consultor internacional en temas de seguridad social, también se ha desempeñado como Jurista en el Departamento de Normas de la Organización Internacional del Trabajo. Correo electrónico: pabloarellano@yahoo.com

Fecha de recepción: 26 de octubre de 2010.

Fecha de aceptación: 15 de diciembre de 2010. 
KEY WORDS: Social Security - Human Rights - ILO - social level Agreement $\mathrm{N}^{\circ} 102$.

\section{INTRODUCCIÓN}

Lamentablemente la crisis económica actual aún no ha mostrado todos los efectos que producirá y sin embargo, estos siguen propagándose más ampliamente y comienzan a afectar a todos. Sin lugar a dudas el mundo no será el mismo después de la crisis y la forma en que concebimos el trabajo y la vida también cambiará. Así el empleo y el trabajo ya no serán vistos de la misma manera. La protección de los desempleados y sus familias se ha convertido en uno de los principales objetivos de los gobiernos en todo el mundo para así lograr el fin de proteger a su población contra los efectos de la crisis. Sin embargo, la protección contra los riesgos sociales no debe ser analizada como una medida temporal en tiempos de crisis.

En este contexto debemos tener en cuenta que actualmente una de cada cinco personas en el mundo tiene algún tipo de prestaciones de seguridad social, y en el contexto de la crisis, esta estimación podría aumentar el número de las personas sin protección. Según Jeff van Langendonck, solo el $20 \%$ de la población mundial goza actualmente de la seguridad social en su verdadero sentido, alrededor del 30\% tienen una cobertura que se está acercando, pero el $50 \%$ restante no se beneficia de ningún dispositivo de las características de la seguridad social ${ }^{1}$. Por su parte, Ursula Kulke, ha señalado que "en 2003, menos del $10 \%$ de la población de los países más pobres se benefician de la protección de la seguridad social adecuada". Además para el mismo ańo, "la cobertura de los regímenes de seguridad social obligatorio en los países en desarrollo con bajos ingresos concierne entre el 10 y el $25 \%$ de la población activa y de personas dependientes de los activos"2.

Las poblaciones no protegidas se encuentran principalmente en el hemisferio sur, en donde los sistemas de seguridad social no están tan desarrollados como los países del hemisferio norte. Estas poblaciones son las más afectadas en el contexto de una crisis global como la actual. Por lo tanto, es de suma importancia construir un sólido sistema de protección de los riesgos sociales, capaz de ofrecer respuestas en un corto, mediano y largo plazo, para evitar los efectos secundarios de las crisis económicas

VAN LANGENDONCK, Jeff (2007). "The meaning of the right to social security", en VAN LANGendonck, Jeff (ed.): The Right to Social Security, Oxford: Intersentia, Antwerp, p. 9.

Kulke, Ursula (2007). "Le rôle actuel et futur des normes de l'OIT dans la mise en œuvre du droit à la sécurité sociale", en Revue internationale de sécurité sociale, vol. 60, 2/3, p. 134. 
en la fuerza laboral, así como también para evitar una crisis social aun más profunda en el futuro. De esta manera será necesario considerar cómo el modelo clásico de la protección fue concebido, mientras que la promoción de un piso de protección social se considera para asignar tal protección.

El Pacto mundial de empleo adoptado por la Conferencia Internacional del Trabajo ${ }^{3}$ de 2009 contiene por primara vez en un instrumento internacional los elementos constitutivos de un piso básico de protección social; este mecanismo puede transformarse, en tiempos de crisis, de una solución temporal de nivel mínimo de protección a una solución permanente para todos. El hecho que se haya incorporado el piso de protección social en el pacto mundial del empleo constituye un reconocimiento de la validez de la iniciativa dentro de la OIT, especialmente en la crisis actual, pero su contenido sigue creando polémica. Por otro lado, en vista de una incorporación más amplia de este mecanismo, cambios en el Estado de bienestar y en los diferentes sistemas de seguridad social nos parece que deben ser estudiados para una mejor apreciación de los pros y contras del piso social.

Debemos añadir que la concepción del piso de protección social constituye un punto de partida de una nueva etapa en la evolución de la seguridad social. Los Estados de bienestar tradicionales basados en sistemas de seguro social o en los regímenes asistenciales universales no han logrado la cobertura para todos. Estos sistemas se desarrollaron en Europa entre 1850 y 1950, pero hoy no es posible aplicar este modelo en países en los que un sector importante de la población activa trabaja en la economía informal. La cual se ha convertido en un importante sector de la economía así como uno de los más desprotegidos. Es evidente que una reestructuración de los modelos de protección clásicos es necesaria y el modelo del Estado del bienestar europeo resulta no ser aplicable en una perspectiva global. Una refundación de la protección de los riesgos sociales otorgada por los Estados debería estudiarse como mecanismo para reformular el Estado de bienestar con el fin de tener una aplicación más amplia. Los sistemas de seguridad social, al mismo tiempo, necesitan una evaluación de sus estrategias de cobertura. La creación de un piso social debe considerar a través en interacción con los mecanismos de seguridad social ya existentes.

Para poder determinar nuevas estrategias de protección y una posible forma de aplicar el piso de protección social proponemos un análisis en tres diferentes niveles. Así analizaremos primero el Estado de bienestar, qué es y sus clasificaciones recientes, para luego ver la seguridad social como un mecanismo sistemático de protección y sus problemas propios.

OIT (2009). Para recuperarse de la crisis: Un Pacto Mundial para el Empleo, adoptado por la Conferencia Internacional del Trabajo, Ginebra, 19 de junio de 2009. 
Finalmente, el piso de protección social en relación a los dos niveles anteriores.

\section{1) El Estado De BiEnestar}

El establecimiento de los principales sistemas de protección social en el periodo de la postguerra mundial le dio a la protección contra los riesgos sociales de un marco institucional cuyo actor principal es el Estado, y he aquí la idea de un Estado de bienestar Pero como subraya la doctrina ${ }^{4}$, el Estado, como una forma de organización de la sociedad, ya no es suficiente para garantizar la protección de sus ciudadanos 5 . Podemos identificar así una de las principales manifestaciones del debilitamiento del Estado en el ámbito de la protección social.

En primer lugar, mientras que el Estado de bienestar, como una forma de organización, se caracteriza por la existencia de una estrecha relación entre la propiedad privada y propiedad colectiva, que da origen a la solidaridad, principio fundamental de la seguridad social y de las relaciones laborales; sin embargo, la seguridad social ha evolucionado hacia una concepción cada vez más individualista. Esto tiende a sustituir el principio de solidaridad y dar a luz a nuevos mecanismos de protección social, como los sistema de seguridad social de administración privada de América Latina o de Europa del Este, que tienden a ser complementados por la creación de mecanismos de protección no contributivos, la tendencia universal, para así cubrir a la población excluida por la concepción individualista ${ }^{6}$.

En segundo lugar, la mutualización de los riesgos en torno al poder público encuentra límites por su incapacidad para extender su protección a nuevas categorías de trabajadores, principalmente en el sector informal o no estructurado. La globalización, mundialización o internacionalización de los mercados ha tenido repercusiones en el mercado laboral y sobre la representación del trabajo. La relación de trabajo tiene nuevas

4 Castel, Robert (2003). L'insécurité sociale. Qu'est-ce qu'être protégé ?, Seuil: La République des Idées.

5 Castel establece una distinción entre la inseguridad de la sociedad civil y la inseguridad social. Nosotros hacemos aquí referencia a la incapacidad estatal de luchar contra la inseguridad en la protección de los riesgos sociales.

6 Esta tendencia se observa actualmente por ejemplo en Bolivia y en Brasil. Ver: CEPALC (2006). La protección social de cara al futuro: acceso, financiamiento y solidaridad, Santiago: CEPALC - Naciones Unidas, p. 120; Barrientos, Armando (2007). "Le rôle de la sécurité sociale financée par l'impôt", en Revue internationale de sécurité sociale, vol. 60, 2/3, p. 111; Bertranou, Fabio (2005). "Los programas del primer pilar en América Latina", en FIAP: El fortalecimiento de los nuevos sistemas provisionales: El rol de cada pilar en la solución del problema de las pensiones, Santiago: Fiap - CIEDESS, p. 57. 
características que por lo tanto requieren de una seguridad social pensada diferentemente. Hay que añadir que la exclusión, de iure o de facto, de ciertas categorías de trabajadores de la seguridad social estatal clásica, de tipo contributiva, basada en el seguro social, hace necesaria la búsqueda de nuevos mecanismos de protección ${ }^{7}$.

Por otra parte, estos cambios no son necesariamente exclusivos de la caracterización del Estado de bienestar. Pero ¿qué es un Estado de bienestar? Retomando la terminología de Titmus ${ }^{8}$, el Esping-Andersen propone una clasificación de tres formas de Estado de bienestar'?

- Los Estados de bienestar liberales (EE.UU., Canadá, Australia), en la que la asistencia se basa en la evaluación de necesidades, transferencias universales modestas y donde los planes de seguro social modestos predominan;

- Los Estados de bienestar socialdemócratas (Europa del Norte), donde existe un dualismo entre Estado y mercado, entre la clase obrera y la clase media y los que se busca una igualdad de más alto nivel en lugar de las necesidades mínimas;

- Los Estados de bienestar corporativista (Alemania, Italia, Francia), conservadores fuertemente ligados al poder público en el que la obsesión liberal con el rendimiento del mercado y la comercialización de mercado nunca es prominente.

La clasificación propuesta se centra en tres características: el rol del Estado, el financiamiento público o privado de las prestaciones y el papel del sector privado. Pero esta clasificación solamente toma en consideración a los países en donde el Estado posee un sistema de seguridad social importante y duradero en el tiempo. Pero podemos utilizar esta terminología y esta clasificación a los países del hemisferio sur y más aún en aquellos de cultura no occidental.

7 Importantes trabajos de han realizado en este sentido por los profesores Filali, Supiot y Deakin.

8 En este sentido ver: Dupeyroux, Jean-Jacques / Borgetto, Michel / Lafore, Robert (2008). Droit de la Sécurité Sociale, 16e édition, Paris: Précis - Dalloz, p. 185; ver también la obra de Titmus, Richard (1958). Essays on the Welfare State, Londres: Allen and Unwin.

9 Esping-Andersen, Gøsta (1999). Les trois mondes de l'Etat-providence, Paris: PUF. Ver también Esping-Andersen Gøsta / Palier, Bruno (2008). Trois leçons sur l'Etat-providence, La république des idées, Paris: Seouil; Esping-Andersen, Gøsta (2007). "The sustainbility of welfare states into the 21st century", en Vij, R. (ed): Globalisation and Werlfare, a critical reader, Palgrave: Macmillan. Esta clasificación ha sido criticada en: Merrien, F-X. / PARChet R. I Kernen, A. (2005). L'Etat social, une perspective internationale, Paris: Armand Colin Collection U. 
Un estudio ${ }^{10}$ ha pretendido responder a la pregunta de si el modelo europeo de Estado de bienestar puede ser exportado. Para ello se simplifica el término Estado de bienestar asimilándolo a un país que consagrara 20 por ciento o más de su PIB a transferencias sociales. El estudio muestra que los países hoy en día en desarrollo gastan más en transferencias sociales que lo que países avanzados de la OECD gastaron históricamente en una etapa similar. Se citan Uruguay, Brasil, Chile y México, como ejemplos de países que cumplen con niveles de gasto avanzados. Sin embargo, ¿estos países poseen un modelo de Estado social comparable al europeo? ¿Las cifras de gasto en PIB sirven para comparar necesidades de culturas distintas? Un estudio que tan solo utiliza el PIB como parámetro de evaluación simplifica demasiado el problema. Si bien el estudio puede ser objeto de críticas por su método de análisis, su autor acierta en todo caso en la afirmación de que el modelo europeo de Estado de bienestar será difícilmente emulado en los países en desarrollo.

En América Latina y Europa del Este, o más aun en países como China o India, en donde está la mayoría de la población mundial queda excluida de esta clasificaciones de Estado de bienestar el concepto europeo parece no encajar por diversas razones de orden local y es justamente en donde las políticas de extensión son más necesarias. Pareciera necesario ampliar el concepto de Estado de bienestar a nuevas culturas, a nuevos horizontes y dejar de considerarlo como un término que hace referencia únicamente a los sistemas de protección social europeos. Se debiera reflexionar en la idea de un Estado como garante de la protección de su población debe ser adaptada a realidades nacionales y regionales fuera del mundo occidental, y además, se deben tener en cuenta las características propias de los mercados laborales.

\section{2) LA SEGURIDAD SOCIAL Y SU CAMPO DE ACCIÓN}

El concepto de seguridad social nace en Europa a fines del siglo XIX y se desarrolló en Europa, América del Norte y Australia, incluso antes de la Segunda Guerra Mundial ${ }^{11}$. Debido a hechos particulares, solo América Latina tuvo un comienzo temprano, en algunos casos antes o durante la Segunda Guerra Mundial, en otras regiones ello ocurre más tarde

10 Lindert, Peter H. (2009). "Can the European Welfare model be exported ?”, en Townsend, Peter (ed): Building decent societies, Rethinking the role of social security in development, ILO, Geneva, Palgrave Macmillan.

11 OIT (1993) Conferencia Internacional del Trabajo, 80ª reunión, El seguro social y la protección social, Memoria del director general (Parte I), Ginebra, OIT, p. 9. 
una vez que se independizaron ${ }^{12}$. Lo cual demuestra por qué la proporción de la población económicamente activa protegida por la seguridad social es más baja en África que en otras partes del mundo, y que general no llega a un 10 por ciento ${ }^{13}$.

Pero, ¿qué es la seguridad social? ¿cuáles son sus objetivos? Hasta ahora tan solo se le ha definido en función de su campo de aplicación: los riesgos sociales clásicos. Si podemos determinar lo que es la seguridad social, sería posible establecer la forma de ampliar la cobertura y los mecanismos para ello. Además es necesario determinar si esos riesgos se pueden incorporar a la noción clásica de seguridad social. Y, por último, debemos determinar si se debe dar un nuevo marco normativo a nivel internacional a la seguridad social.

\section{1) LA CONSAGRACIÓN DE LA SEGURIDAD SOCIAL}

La denominación, así como la idea, de un derecho de la seguridad social como una rama independiente del derecho es reciente. La consagración ha seguido de una lenta progresión a nivel internacional, proceso que comprende la búsqueda de una definición como rama del Derecho y el desarrollo de su contenido. Es la ley federal de los Estados Unidos "Social Security Act" de 1935 el primer paso en este sentido. Sin embargo, son sobre todo el informe de Sir William Beveridge ${ }^{14}$ y de los resultados de la Conferencia Internacional del Trabajo en Filadelfia ${ }^{15}$ que marcaron el comienzo de una importante extensión de la idea de la seguridad social. El concepto fue desarrollado en los países industrializados durante el período de entreguerras y que conoció un nuevo enfoque como un derecho humano universal gracias a los instrumentos de las $\mathrm{Na}$ ciones Unidas.

Esta rama toma forma gracias a la Declaración Universal de los Derechos Humanos que fue adoptada por la Asamblea General de las Naciones Unidas el 10 de diciembre de $1948^{16}$, por 48 votos a favor y 8 abstenciones (correspondientes a los votos de los antiguos países comunistas),

\footnotetext{
12 Rys, Vladimir (2010). Reinventing social security worldwide, Back to the essentials, Londres: The Policy Press.

13 OIT (1993) 33.

14 Beveridge, William (1942). Social insurance and allied services, London: HM Stationery Services. Ver también: Dupeyroux / Borgetto / Lafore (2008) 34 y ss.

15 Adopción por la OIT de la Declaración de Filadelfia y de las Recomendaciones 67 y 69.

16 La Declaración Universal de los Derechos del Hombre fue adoptada y proclamada por resolución de la Asamblea General de Naciones Unidas 217 A (III) el 10 de diciembre de 1948. Ver: Chagnollaud, Dominique / Drago, Guillaume (sous la direction de) (2006). Dictionnaire des droits fondamentaux, Paris: Dalloz, pp. 206 y ss.
} 
en sus artículos 22 y 25 . En el artículo $22^{17}$, la Declaración ofrece un mayor ámbito de protección incluyendo no solo los trabajadores del sector privado sino que también a toda persona como miembro de la socie$\mathrm{dad}^{18}$. Este derecho es expresado como un derecho universal. El artículo $25^{19}$ por su lado sugiere por tanto dos niveles de protección: el establecimiento de un nivel adecuado de vida, de aplicación general, y el derecho a una protección particular contra determinadas contingencias. Además, según el profesor Jeff van Langendonck, este artículo sirve como una definición de la seguridad social, ya que contiene una lista de riesgos a cubrir para el conjunto de los beneficiarios (aunque este autor señala que la protección contra el riesgo de viudez aparece concernir solamente las mujeres).

La Declaración se presenta como un ideal común a alcanzar en nombre de una concepción común de los derechos humanos y las libertades fundamentales ${ }^{20}$, entre los cuales, el derecho a la seguridad social fue concebido en un espíritu de progreso social en relación con el período económico especial de la reconstrucción de la postguerra. Es en este contexto que se debe entender la idea de un derecho a la seguridad social concebido como derecho universal. Considerar este derecho como un derecho fundamental, que todos los ciudadanos puedan disfrutar de la misma manera era una idea nueva, para los firmantes de la Declaración ${ }^{21}$. Sin embargo, en esa misma época, es la técnica del seguro social que ha primado durante la puesta en marcha de este derecho. Ahora bien, esta técnica fue concebida para los trabajadores, y aún más restrictivamente: para los trabajadores de empresas privadas. Así pues, esta técnica no fue capaz de asegurar la concretización de la vocación universalista original del derecho a la seguridad social. He aquí uno de los principales motivos del por qué la seguridad social no ha logrado el objetivo de la cobertura universal: mecanismo con un alcance subjetivo limitado.

17 "Toda persona, como miembro de la sociedad, tiene derecho a la seguridad social, y a obtener, mediante el esfuerzo nacional y la cooperación internacional, habida cuenta de la organización y los recursos de cada Estado, la satisfacción de los derechos económicos, sociales y culturales, indispensables a su dignidad y al libre desarrollo de su personalidad".

18 Dupeyroux, Jean-Jacques (1960). "Quelques réflexions sur le droit à la sécurité sociale", en Dr. Soc. p. 293.

19 "Toda persona tiene derecho a un nivel de vida adecuado que le asegure, asi como a su familia, la salud y el bienestar, y en especial la alimentación, el vestido, la vivienda, la asistencia médica y los servicios sociales necesarios; tiene asimismo derecho a los seguros en caso de desempleo, enfermedad, invalidez, viudez, vejez u otros casos de pérdida de sus medios de subsistencia por circunstancias independientes de su voluntad. La maternidad y la infancia tienen derecho a cuidados y asistencia especiales. Todos los niños, nacidos de matrimonio o fuera de matrimonio, tienen derecho a igual protección social".

20 Decaux, E. (1998). "Les droits fondamentaux en droit international" en AJDA, p. 66.

21 VAN LANGendonck (2007) 4. 
Por su lado, el Pacto Internacional de Derechos Económicos, Sociales y Culturales de $1966^{22}$ marca una segunda etapa hacia el establecimiento del derecho a la seguridad social como un derecho universal ${ }^{23}$ y es un instrumento complementario a la Declaración ${ }^{24}$. El Pacto, en el artículo 9 establece que: "Los Estados Partes en el presente Pacto reconocen el derecho de toda persona a la seguridad social, incluso al seguro social". Esta norma ha sido criticada porque no indica la naturaleza o el nivel de protección a asegurar. Pero como Riedel señala el concepto de seguridad social se ha dejado abierto explícitamente ${ }^{25}$ con el fin de incluir provisiones de naturaleza diferente así como para incluir a la asistencia social ${ }^{26}$.

\section{2) EL MODELO DE BASE}

El modelo que inspiró a la concepción de la seguridad social se llama "menbreading" o del hombre "breadwinner", según el cual es el hombre que trabaja a tiempo completo y aporta la comida a su familia, la mujer se queda en casa para administrar el hogar y cuidar a los niños ${ }^{27}$. El derecho a la seguridad social se desarrolló en un contexto de postguerra en donde predominaba el trabajo en Europa y en donde el trabajador masculino fue considerado como un beneficiario directo de este derecho ${ }^{28}$. De hecho, los primeros convenios de la OIT en materia de seguridad social son convenios para los hombres, en los cuales las mujeres son a menudo descrito como "la mujer de" 29 .

22 El Pacto Internacional de Derechos Económicos, Sociales y Culturales adoptado por resolución de la Asamblea General 2200 A (XXI) del 16 diciembre de 1966. Ver: Chagnollaud / Drago (2006) 206 y ss.

23 Ver: Langford, Malcolm (2007). "The Right to Social Security and Implications for Law, Policy and Practice" en Riedel, Eibe (dir.): Social Security as a Human Right. Drafting a general comment on article 9 ICESCR-Some challenges, Berlin: Springer, p 29; LAMARCHE, Lucy (2002). "The right to social security in the international covenant on economic, social and cultural rights", en Chapman, Audrey / Rusell, Sage (eds.): Core Obligations: Building a Framework for Economic, Social and Cultural Rights, Oxford- New-York: Intersentia - Antwerp, p 87; Dupeyroux, J. J. (1960 b). "Le droit à la sécurité sociale dans les déclarations et pactes internationaux" en Dr. Soc. p. 365.

24 Ver: Lamarche (2002) 87.

25 Un intento por definir el concepto de la seguridad social se hizo con ocasión de la Primera Conferencia Interamericana sobre Seguridad Social, en Santiago de Chile en 1942, ver Perrin, Guy (1969). "Reflections on fifty years of social security" en International Labour Review, Vol. 99, No 3, p. 249.

26 Riedel, Eibe (2007). "The Human Right to Social Security. Some challenges", en Riedel, Eibe (dir.): Social Security as a Human Right. Drafting a general comment on article 9 ICESCR-Some challenges, Berlin: Springer, p. 23.

Ver: EsPING ANDERSEN (2007) 52.

LAMARCHE (2002) 90.

LAMARCHE (2002) 92. 
La acción normativa de la OIT comienza desde el preámbulo de su Constitución, aprobada en $1919^{30}$, con una protección contra los riesgos sociales a partir de la técnica del seguro social, que señala: "lucha contra el desempleo (...), protección del trabajador contra las enfermedades, sean o no profesionales, y contra los accidentes del trabajo, protección de los niños, de los adolescentes y de las mujeres, pensiones de vejez y de invalidez...". Por su lado el Convenio No 102 sobre norma mínima, Convenio de referencia en este tema, es esencialmente un compromiso entre el sistema de Bismarck de seguros sociales reservado a los trabajadores y el modelo de Beveridge de la seguridad social universal. Para este Convenio, el modelo de bredwinner es una ficción que permite determinar el monto de las prestaciones en todos los países y así poder establecer un punto de comparación.

Sin embargo este modelo ha evolucionado hoy en día no reviste de las mismas características que tenía en los años cincuenta. El mercado laboral ha cambiado, un gran número de mujeres trabajan y representan una fuente de ingresos para sus familias, sin contar las mujeres que crían solas a sus hijos ${ }^{31}$. Esta idea se refuerza en los dichos del propio Director General de la OIT en 1993 quien señalaba que "las prestaciones de desempleo establecidas en los decenios de 1950 y 1960 presuponían una estructura familiar -familia nuclear a cargo de un cabeza de familia, de sexo masculino- cada vez más anacrónica, debido a la proporción creciente de mujeres (casadas o no) que se incorporaban a la población activa y al número cada vez mayor de divorcios, con el aumento consiguiente del número de familias monoparentales"32. Además, el modelo de "breadwinner" de los países europeos no se ha propagado, como que se esperaba, a los países en desarrollo. Según algunos, esto se manifiesta por el hecho de que las disposiciones del Convenio 102 pertenecen a un modelo industrial que no corresponde ni a los países llamados desarrollados ni a aquellos países llamados en vías de desarrollo ${ }^{33}$. A lo que debemos agregar la economía informal, que ha crecido fuertemente en muchos países en desarrollo ${ }^{34}$, lo que sugiere que el mercado laboral ha

30 Constitución de la Organización International del Trabajo, Tratado de Versailles, Parte XIII, 1919.

31 Pennings, Frans / Schulte, Bernd (2006). "Some current points of discussion concerning social security conventions", en Pennings, Frans (ed.): Between Soft and Hard Law. The impact of International social Security Standards on National Social Security Law, Studies in Employment and Social Policy, The Hague: Kluwer Law International, p. 45. Ver también: LAMARCHE (2002) 90.

32 OIT (1993) 75

33 Supiot, Alain (2006). "Esquisse d'un accord-cadre relatif à l'extension de la protection sociale" en Semaine Sociale Lamy Supplément, No 4, septiembre, No 1271, p. 91.

34 Reynaud, Emmanuel (2007). "The right to social security - Current challenges in international perspective", en Riedel, Eibe (dir.): Social Security as a Human Right. Drafting a general comment on article 9 ICESCR-Some challenges, Berlin: Springer, p. 1. 
evolucionado y que la estructura de este mercado es diferente en estos dos grupos de países.

La seguridad social, rama sin definición pero con un campo de cobertura explicitado, no ha podido en su concepción más clásica, con sus mecanismos de asistencia social y de seguros sociales, alcanzar el objetivo de la cobertura universal. Se abre la puerta a una nueva evolución en lo que se refiere a la protección contra los riesgos sociales: una nueva seguridad social, ya no basada en los mecanismos clásicos.

\section{3) EL PISO DE PROTECCIÓN SOCIAL PUNTO DE CONVERGENCIA HA- CIA UNA NUEVA FORMA DE PROTECCIÓN}

La cobertura universal es una necesidad global, pero cómo ponerlo en práctica es una estrategia nacional. Para establecer esta estrategia, los dos ejes antes mencionados deben tenerse en cuenta para una política pública específica. Así, en los años cincuenta la discusión sobre la adopción de un convenio sobre la seguridad social giraba en torno a dos instrumentos, uno de una Norma Mínima y otro que contendría una norma avanzada. Durante la Conferencia Internacional del Trabajo de 1952, por falta de tiempo, tan solo se logró discutir y adoptar el convenio de la norma minima, Convenio No. 102.

Los convenios de la OIT tienen un rol importante en la legislación nacional desde el momento que ellos son ratificados por un país. Pero los convenios de la OIT sobre la seguridad social tienen una tasa de ratificación más baja que aquellos relativos al derecho del trabajo ${ }^{35}$. Sin embargo, debemos recalcar la paradoja que si bien el Convenio No.102 fue concebido como la norma mínima en realidad ha llegado a convertirse en un convenio que contiene exigencias consideradas erróneamente altas para muchos países y no ratificados; y a su vez muchos países cumplen con los niveles mínimos del convenio y no lo ratifican por simple desconocimiento de su contenido.

Recientemente, la OIT ha presentado el fortalecimiento de la seguridad social como uno de los cuatro objetivos estratégicos de la Agenda de Trabajo Decente, presentada por el Director General y aprobados por la Conferencia Internacional del Trabajo en 1999. En la misma línea, los países miembros reafirmaron el papel fundamental de la OIT en la promoción y extensión de la seguridad social, así como sus obligaciones en este ámbito, durante la Sesión de 2001 de la Conferencia Internacional del Trabajo. En el 2003, se lanzan dos campańas: una para promover la extensión de la cobertura de la seguridad social, y la otra, la cobertura

35 LANGFORD (2007) 31. 
para todos. Así, se ha llegado en la OIT a la proposición de un piso social como solución al problema de la extensión, como se le ha denominado, y que no es otra cosa que el de la cobertura universal prevista en las normas internacionales de los años cincuenta.

El piso es esencialmente un grupo de garantías de transferencias sociales en dinero o en especie. Se ha formulado como un grupo de garantías y no como un grupo definido de prestaciones o de derechos. Además se deja la opción abierta a los países para que cada uno concretice estas garantías en la forma de prestaciones condicionadas o universales. Lo esencial es el hecho de que toda persona tenga acceso a estas trasferencias esenciales. Los expertos de la OIT han hecho saber que consideran a este concepto como potencialmente central para un instrumento que dé efecto al derecho humano a la seguridad social ${ }^{36}$, aun cuando ya existen instrumentos que cumplen este objetivo.

El piso de protección social ha sido conceptualizado por la OIT en el Pacto Mundial para el Empleo adoptado por la Conferencia Internacional del Trabajo en $2009^{37}$. En este texto se solicita a los países que aún no han extendido todavía la seguridad social de construir una "adecuada protección social para todos, basada en un piso básico de protección social, que incluya:

36 OIT (2009). Etendre la sécurité sociale à tous: Tour d'horizon des difficultés, des pratiques actuelles et des options stratégiques. Document préliminaire pour discussion, Réunion tripartite d'experts sur les stratégies d'extension de la couverture de sécurité sociale, Bureau international du Travail, Département de la Sécurité Sociale, Genève, BIT, 2-4 de septiembre de 2009. Ver además: Behrendt, Christina et al. (2009). "Rethinking the role of social security in development", en Townsend, Peter (ed): Building decent societies, Rethinking the role of social security in development, ILO, Geneva, Palgrave Macmillan.

37 La noción de piso de protección social también ha sido adoptada por el sistema de Naciones Unidas, pero aquí su contenido toma un campo muchísimo mas amplio, alejándose de la idea de base de la OIT y sobre todo de una protección basada en derecho. En abril 2009, el UN Chief Executives Board (CEB) acordó nueve iniciativas conjuntas para enfrentar a la crisis, acelerar la recuperación y pavimentar el camino para una globalización más justa y sostenible: Financiación adicional para los más vulnerables; Seguridad alimentaria; Comercio; Una Iniciativa por una Economía Verde; Un Pacto Global por el Empleo; Un Piso de Protección Social; Seguridad humanitaria y estabilidad social; Tecnología e Innovación; y, Monitoreo y Análisis. Asi el contenido del Piso de Protección Social dentro de la iniciativa de la ONU abarca un conjunto de derechos sociales, infraestructuras y servicios básicos de los cuales deberían beneficiarse todos los ciudadanos del mundo. El piso ha de concebirse como la obligación fundamental de velar por la realización de un nivel mínimo elemental de esos derechos, consagrados en los tratados de los derechos humanos. El piso de protección social consistiría en dos elementos principales que pueden contribuir al ejercicio efectivo de esos derechos: 1.- Asegurar la disponibilidad, la continuidad y la accesibilidad geográfica y financiera de servicios esenciales tales como agua potable, saneamiento, alimentos y nutrición adecuada, salud, educación, vivienda y otros servicios sociales relativos a seguros de vida y ahorro. 2.- Asegurar la demanda efectiva mediante la garantía de un conjunto básico de transferencias sociales, en metálico o en especie, que brinde un ingreso y medios de subsistencia mínimos a la población pobre y vulnerable y que facilite el acceso a los servicios esenciales. Esto incluye transferencias sociales (además de información, derechos y políticas) destinadas a los niños, a las personas en edad activa con ingresos insuficientes y a las personas de edad avanzada. 
- Acceso a asistencia médica,

- Seguridad de los ingresos para los ancianos y para personas con discapacidades,

- Prestaciones para los hijos a cargo, y

- Seguridad de los ingresos combinada con esquemas de garantías"38.

Creemos sinceramente que la OIT se encuentra en buen camino pero sus propuestas deben ser afinadas aun más. Creemos que existe una confusión terminológica que nos gustaría aclarar; y además llamar la atención sobre los actuales mecanismos de extensión que se promocionan dentro del marco de las campañas de extensión del piso social. Hoy en día, ya no se habla de seguridad social, se habla más bien de protección social. Pero, ¡en qué consiste esta última? La protección social comúnmente incluye al seguro social, a la asistencia social y a regulaciones del mercado de trabajo ${ }^{39}$. La seguridad social desde sus inicios fue concebida como una rama que incluía dos tipos de mecanismos de cobertura: el seguro social y la asistencia social, así se vio reflejado en el Convenio No. 102 de la OIT. La protección social incluye otro tipo de medidas de regulación del mercado de trabajo que a su vez tienden a proteger pero ya no por medio de los mecanismos de la seguridad social, utiliza lo que se conoce como políticas de activación. Tanto la seguridad social como la protección social tienen el mismo objeto: proteger riesgos sociales. Sin embargo, el piso social pertenece a la protección social y no a la seguridad social, en su defecto se encuentra en una zona gris y limítrofe. Esto último debido a que muchos de los programas que se utilizan como ejemplo del piso social revisten características propias de un mecanismo de asistencia social, y por tanto pertenecientes de seguridad social. Pero se trata de una nueva asistencia social con características nuevas distintas a la asistencia social clásica, ya que sus prestaciones son focalizadas y condicionadas: hablamos entonces de un asistencialismo acentuado.

El piso social ha sido promovido dando como ejemplo una serie de programas de protección social que se han catalogado bajo la categoría de transferencias monetarias condicionadas. Así un número importante de países en vías de desarrollo han implementado programas que tienden a la extensión de la seguridad social a través de estas transferencias condicionadas, fenómeno particularmente interesante en Latinoamérica (Brasil, Argentina, México) así como también en África (prestaciones familiares en Sudáfrica). Los programas de transferencias condicionadas sin duda al-

38 OIT (2009). Para recuperarse de la crisis: Un Pacto Mundial para el Empleo, adoptado por la Conferencia Internacional del Trabajo, Ginebra, 19 de junio de 2009.

39 Barrientos, Armando (2009). "Introducing basic social protection in low-income countries: Lessons from existing programmes" en Townsend, Peter (ed): Building decent societies, Rethinking the role of social security in development, ILO, Geneva, Palgrave Macmillan. 
guna han logrado aumentar la cobertura en regiones en donde la informalidad es alta y en donde los mecanismos de seguros sociales no han podido ampliar su cobertura. Sin embargo, desde una perspectiva de los derechos que estos programas pueden otorgar nos encontramos frente a un vacío.

Desde sus orígenes la OIT se ha caracterizado por su elemento normativo como la base de su actuar. Tanto los convenios como las recomendaciones, para aquellos que opten por ellas, contienen una norma que define como las legislaciones de los países deben otorgar derechos con consagración legal a sus poblaciones. Los programas de transferencias condicionadas no siempre van acompañados de su consagración legal, se trata de meros programas del gobierno de turno. Pero más aun, estos programas dependen de los donadores y también de la partida presupuestaria para el año en que se ejecuten. Por tanto, no existe un derecho adquirido sobre la prestación. Si el programa desaparece en un cambio de gobierno no existe un mecanismo jurídico de hacer exigible el derecho. Resulta interesante recalcar que la OIT promociona un mecanismo, que si bien extiende la cobertura social y lo aplaudimos, no otorga derechos al público destinatario lo que contradice los objetivos de la propia organización. Más aun, las conclusiones de la Reunión tripartita sobre las estrategias de extensión de la cobertura de la seguridad social ${ }^{40}$ sugieren que los actores sociales se pondrán de acuerdo sobre un instrumento no vinculante: una recomendación. Un instrumento de la OIT definirá los elementos del piso de protección de base así como una estrategia para adoptarlo. Sin embargo, el camino para la adopción de la recomendación aún es largo y su efecto no vinculante deja dudas con respecto a la capacidad de otorgar derechos y extender la protección. De acuerdo a las últimas evoluciones en torno al piso social pareciera que el futuro instrumento que lo consagraría seria un instrumento de orden político y no normativo.

\section{COMENTARIOS FINALES}

Como hemos vistos las nociones de Estado de bienestar y de seguridad social tienen su origen en evoluciones europeas que influencian al resto de las regiones del mundo. Pero hoy en día, los análisis muestran que este camino ha sido el equivocado. Intentar aplicar el Estado de bienestar europeo en otras regiones no tiene sentido y los mecanismos de seguridad social tradicionales ha mostrado ser ineficaces en regiones con alta informalidad. Se hace necesario un mecanismo que comprenda las realidades propias de cada región evitando aplicar de manera forzada el modelo europeo. El piso de protección social propuesto por la OIT se

$40 \quad$ Ver: OIT (2009). 
presenta con un instrumento alternativo que permitiría alcanzar el objetivo de la cobertura universal. Sin embargo este no debe ser implementado sin tener en cuenta los mecanismos de seguridad social existentes y de la concepción del Estado de bienestar en un país determinado. Para implementar este piso social resulta imprescindible poder clarificar los dos niveles ya mencionados sin tener en cuenta las influencias europeas. Debe primar la realidad regional y local.

El Convenio No 102 fue concebido como la norma mínima y la idea de la norma avanzada no fue adoptada en 1952: hoy en día el convenio 102 se ha convertido en la norma avanzada a la cual los países difícilmente logran alcanzar, por un nivel bajo de desarrollo o por simple desconocimiento. Una norma con carácter vinculante se hace cada vez más necesaria para proteger a la inmensa mayoría que no posee ninguna prestación. La discusión hoy se centra en saber si esta norma debería contener los elementos del piso de protección social de base. Si este fuese el caso esta norma debe poseer al menos tres características: que posea una flexibilidad más grande aun que el Convenio No 102 para que se adapte en todas las regiones del mundo; que tome en cuenta la diversidad de los Estados y de sistemas de seguridad social; y, que otorgue derechos a los destinatarios. Pero, por otro lado, la OIT debería estudiar con mayor profundidad la promoción del Convenio 102, cuyos mecanismos siguen vigentes y que incluso pueden contener los elementos del piso social. Para que crear otra norma internacional si el Convenio 102 otorga derecho y contiene las garantías básicas. La decisión hoy en día está en manos de los delegados de la próxima Conferencia Internacional del Trabajo que discutirá el tema en junio de 2011.

La historia muestra que es en los periodos posteriores a las crisis cuando se efectúan las grandes reformas, así la OIT nace en el Tratado de Versalles y la seguridad social nace después de la Segunda Guerra Mundial. Como hemos expuesto, actualmente nos enfrentamos a una profunda transformación en los mecanismos de protección de riesgos sociales pasando de una lógica basada en la consagración de derechos (como el Convenio No 102) a una basada en políticas sociales de acceso a prestaciones condicionadas (piso social). A nuestro parecer una refundación de la protección debe tener en cuenta los tres niveles de protección de los riesgos sociales expuestos creando una articulación más profunda entre ellas y que se base en el tratamiento de derechos.

\section{BIBLIOGRAFÍA}

- Barrientos, Armando (2009). "Introducing basic social protection in low-income countries: Lessons from existing programmes" en Townsend, Peter (ed.): Building decent societies, Rethinking the role of social security in development, ILO, Geneva, Palgrave Macmillan. 
(2007). "Le rôle de la sécurité sociale financée par l'impôt" en Revue Internationale de Sécurité Sociale, vol. 60, 2/3, p. 111.

- Behrendt, Christina et al. (2009). "Rethinking the role of social security in development" en Townsend, Peter (ed): Building decent societies, Rethinking the role of social security in development, ILO, Geneva, Palgrave Macmillan.

- Bertranou, Fabio (2005). "Los programas del primer pilar en América Latina", en FIAP: El fortalecimiento de los nuevos sistemas provisionales: El rol de cada pilar en la solución del problema de las pensiones, Santiago: Fiap - CIEDESS.

- Beveridge, William (1942). Social insurance and allied services, Londres: HM Stationery Services.

- Castel, Robert (2003). L'insécurité sociale. Qu'est-ce qu'être protégé ?, Paris: La République des Idées - Seuil.

- CEPALC (2006). La protección social de cara al futuro: acceso, financiamiento y solidaridad, Santiago: CEPALC - Naciones Unidas.

- Chagnollaud, Dominique / Drago, Guillaume (sous la direction de) (2006). Dictionnaire des droits fondamentaux, Paris: Dalloz.

- Decaux, E. (1998). "Les droits fondamentaux en droit international" en $A J D A$, p. 66.

- Dupeyroux, Jean-Jacques (1960). "Quelques réflexions sur le droit à la sécurité sociale" en Dr. Soc. p. 293.

(1960 b) "Le droit à la sécurité sociale dans les déclarations et pactes internationaux", Dr. Soc. p. 365.

- Dupeyroux, Jean-Jacques / Borgetto, Michel / Lafore, Robert (2008). Droit de la Sécurité Sociale, 16e édition, Paris: Précis Dalloz.

- Esping Andersen, Gøsta (1999). Les trois mondes de l'Etat-providence, Paris: PUF.

(2007). "The sustainbility of welfare states into the 21 st century" en Vij, R. (ed): Globalisation and Werlfare, a critical reader, Palgrave Macmillan

- Esping-Andersen Gøsta / Palier, Bruno (2008). Trois leçons sur l'Etat-providence, La république des idées, Paris: Seuil.

- Kulke, Ursula (2007). "Le rôle actuel et futur des normes de l'OIT dans la mise en ouvre du droit à la sécurité sociale" en Revue internationale de sécurité sociale, vol. 60, 2/3, p. 134.

- Lamarche, Lucy (2002). "The right to social security in the international covenant on economic, social and cultural rights" en Chapman, Audrey / Rusell, Sage (eds.): Core Obligations: Building a Framework for Economic, Social and Cultural Rights, Oxford- New-York - Antwerp: Intersentia.

- Langford, Malcolm (2007). "The Right to Social Security and Implications for Law, Policy and Practice" en Riedel, Eibe (dir.) Social 
Security as a Human Right. Drafting a general comment on article 9 ICESCR-Some challenges, Berlin: Springer.

- VAN LANGENDONCK, Jeff (2007). "The meaning of the right to social security”, en van Langendonck, Jeff (ed.). The Right to Social Security, Oxford - Antwerp: Intersentia.

- Merrien, F-X. / Parchet R. / Kernen, A. (2005). L'Etat social, une perspective internationale, Paris: Armand Colin Collection U.

- Lindert, Peter H. (2009). "Can the European Welfare model be exported?" en Townsend, Peter (ed): Building decent societies, Rethinking the role of social security in development, ILO, Geneva, Palgrave Macmillan.

- OIT (2009). Etendre la sécurité sociale à tous: Tour d'horizon des diffcultés, des pratiques actuelles et des options stratégiques. Document préliminaire pour discussion, Réunion tripartite d'experts sur les stratégies d'extension de la couverture de sécurité sociale, Bureau international du Travail, Département de la Sécurité Sociale, Genève, BIT, 2-4 de septiembre de 2009.

- OIT (2009). Para recuperarse de la crisis: Un Pacto Mundial para el Empleo, adoptado por la Conferencia Internacional del Trabajo, Ginebra, 19 de junio de 2009.

- OIT (1993). Conferencia Internacional del Trabajo, 80a reunión, El seguro social y la protección social, Memoria del director general, (Parte I), Ginebra, OIT.

- Pennings, Frans / Schulte, Bernd (2006). "Some current points of discussion concerning social security conventions" en Pennings, Frans (ed.): Between Soft and Hard Law. The impact of International social Security Standards on National Social Security Law, Studies in Employment and Social Policy, The Hague: Kluwer Law International.

- Perrin, Guy (1969). "Reflections on fifty years of social security" en International labour review, Vol. 99, No 3, p. 249.

- Reynaud, Emmanuel (2007). "The right to social security - Current challenges in international perspective" en Riedel, Eibe (dir.): Social Security as a Human Right. Drafting a general comment on article 9 ICESCR-Some challenges, Berlin: Springer.

- Riedel, Eibe (2007). "The Human Right to Social Security. Some challenges", en Riedel, Eibe (dir.): Social Security as a Human Right. Drafting a general comment on article 9 ICESCR-Some challenges, Berlin: Springer.

- Rys, Vladimir (2010). Reinventing social security worldwide, Back to the essentials, Londres: The Policy Press.

- Supiot, Alain (2006). "Esquisse d'un accord-cadre relatif à l'extension de la protection sociale" en Semaine Sociale Lamy Supplément, 4 septembre, No 1271, p. 91

- Titmus, Richard (1958) Essays on the Welfare State, Londres: Allen and Unwin. 